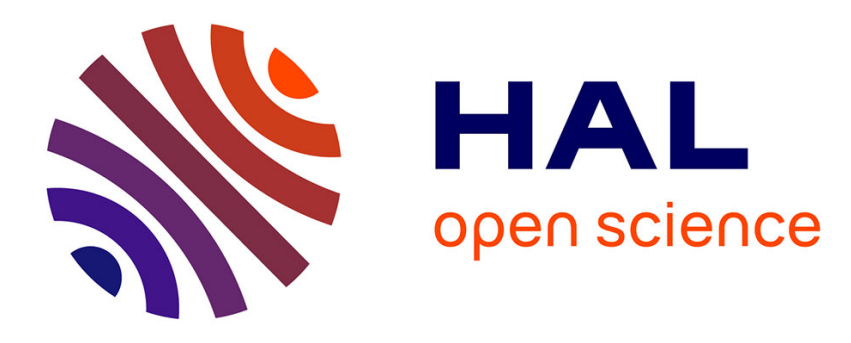

\title{
Combination of Sources of Evidence with Distinct Frames of Discernment
}

\author{
Yilin Dong, Xinde Li, Jean Dezert
}

\section{To cite this version:}

Yilin Dong, Xinde Li, Jean Dezert. Combination of Sources of Evidence with Distinct Frames of Discernment. FUSION 2018, Jul 2018, CAMBRIDGE, United Kingdom. pp.2513-2520, 10.23919/ICIF.2018.8455451 . hal-02335345

\section{HAL Id: hal-02335345 \\ https://hal.science/hal-02335345}

Submitted on 28 Oct 2019

HAL is a multi-disciplinary open access archive for the deposit and dissemination of scientific research documents, whether they are published or not. The documents may come from teaching and research institutions in France or abroad, or from public or private research centers.
L'archive ouverte pluridisciplinaire HAL, est destinée au dépôt et à la diffusion de documents scientifiques de niveau recherche, publiés ou non, émanant des établissements d'enseignement et de recherche français ou étrangers, des laboratoires publics ou privés. 


\section{Combination of Sources of Evidence with Distinct Frames of Discernment}

\author{
Yilin Dong \\ Key Laboratory of Measurement \\ and Control of CSE \\ School of Automation \\ Southeast University \\ Email:dyl@seu.edu.cn
}

\author{
Xinde Li \\ Corresponding Author \\ Key Laboratory of Measurement \\ and Control of CSE \\ School of Automation, Southeast University \\ Email: xindeli@seu.edu.cn
}

\author{
Jean Dezert \\ The French Aerospace Lab \\ ONERA \\ DTIM/MSDA \\ F-91123 Palaiseau, France. \\ Email:jean.dezert@onera.fr
}

\begin{abstract}
Multi-source information fusion strategies in target recognition have been widely applied. Generally, each source is defined and modelled over a common frame composed of the hypotheses to discern. However, in practice, the independent sources of evidence can refer to distinct frames of discernment in terms of the hypotheses they consider. Under this condition, the classical combination process cannot be applied directly. Working with distinct frames of discernment for information fusion is a problem often encountered in the development of recognition systems which requires a particular attention. In order to combine such sources, this paper presents a new combination method which splits the process of fusion into two steps: construction of granular structure, calculation of belief mass, followed by the fusion process. Our simulations results show that the proposed method can effectively solve the problem of fusion of sources defined on distinct frames.
\end{abstract}

Keywords: Information fusion, Distinct frames of discernment, Belief functions, Combination Rule, Granular Structure, Rough set.

\section{INTRODUCTION}

The aim of this paper is to propose a new approach for combining sources of evidence referring to distinct (uncommon) frame of discernment, that can be eventually (partially) incompatible.

\section{Motivation}

In Dempster-Shafer Theory (DST) also known as the theory of evidence [1], each Source of Evidence (SoE) refers to a (common) Frame of Discernment (FoD) with exhaustive and exclusive elements. Each element of the FoD corresponds to a choice for the solution of the problem under concern. In some applications, the FoD related with a source is not always exhaustive and exclusive and that is why Dezert-Smarandache Theory (DSmT) [2] has been developed. Moreover, in more realistic situations, the FoDs related with each source can be distinct so that no global common FoD is directly available in order to apply classical fusion techniques developed in DST or DSmT. The fusion of sources of evidence related to distinct FoDs encountered in real applications motivates the works presented in this paper.

\section{State of the art and challenges}

In order to fuse sources of evidence referring to distinct FoDs, some scholars have proposed different methods. The most representative ones are presented in [3], [4], [5] and [6]. These methods can be classified into three categories as follows.

1) Expanded methods: In these methods, for example in [3], a common (expanded) FoD is built and each $\mathrm{SoE}$ assigns a zero mass value to elements of this common FoD that are not included in its own specific FoD. This kind of method is simple and effective up to some degree. The implicit assumption done in these methods is that each source of evidence can identify the expanded common FoD as being the disjunction of all own specific FoDs of the sources. This implicit assumption is however unreasonable because it overlooks the main fact that the elements of the expanded common FoD are not within the scope of expertise of each source under concern.

2) Deconditioning methods: To overcome the problem of expanded methods, Janez [3] adopted a new standpoint, and he developed a deconditioning fusion method. This method can be interpreted as the opposite (dual) of the extension method. The so-called discriminable domain is divided according to the FoD of each source of evidence. For example, we consider two SoE $S_{1}$ and $S_{2}$ with FoDs $\Theta_{1}$ and $\Theta_{2}$ and let $^{1} \Theta_{c} \triangleq \Theta_{1} \cap \Theta_{2}$ be the common part of $\Theta_{1}$ and $\Theta_{2}$ (assumed to be non empty). The elements of $\Theta_{1} \cap \bar{\Theta}_{c}$ will be discriminated only by source $S_{1}$, whereas the elements of $\Theta_{2} \cap \bar{\Theta}_{c}$ will be discriminated only by the $S_{2}$. The elements of $\Theta_{c}$ will be discriminated by both sources $S_{1}$ and $S_{2}$. Such kind of separation is based on the range of each evidence, which may yield to some ambiguities of the extension methods. This separation principle splits the original fusion process into three sub-steps, in which one needs to make the fusion. Hence, with these methods one can degrade the computational efficiency of the fusion strategy, especially if large databases have to be browsed.

3) Conditioning updating methods: Unlike the previous methods, the more recent conditional method proposed by Premaratne et al. in [4], [5] and [6], is a different approach, which does not need to expand the FoDs of each source into

\footnotetext{
${ }^{1}$ Here, we use the symbol $\triangleq$ to mean equal by definition.
} 
a common FoD, nor to separate and identify the common elements of FoDs. In this new (conditioning and updating) method each source updates its knowledge base by selecting the incoming evidence that it can discern (or it is interested in). The performance of such method is however very dependent on the ad-hoc choice of two weighting tuning parameters.

\section{Contribution}

As aforementioned discussed, the existing methods have all their own limitations and drawbacks. In this paper, we propose to combine the granular computing developed by Pawlak [7] in his Rough Set Theory (RST) and belief mass allocation in Belief Functions (BFs). Specifically, we split the process of fusion into two steps: 1) the construction of granular structure (GS) based on the defined relations, and 2) the calculation of belief mass of target focal element (TFE). The innovation of GS construction is that at first we determine the indistinguishability relation according to TFE. Then we construct the relevant GS about the indistinguishability relation in the framework of the available FoDs. The calculation of the belief mass of TFE is the summation of the related masses of TFE in each GS with conflict redistribution, followed by a normalization step.

This paper is organized as follows. Section 2 reviews some basic concepts of belief functions. Section 3 discusses fusion strategy based on RST for SoE defined on distinct FoDs. Section 4 gives the summary of the new proposed combination method. In section 5, a target identification case is chosen to show the performance of this new approach. Section 6 concludes the paper and proposes some perspectives.

\section{BASICS OF BELIEF FUNCTIONS}

In DST framework [1], the FoD $\Theta \triangleq\left\{\theta_{1}, \ldots, \theta_{n}\right\}(n \geq 2)$ is a set of exhaustive and exclusive elements. This is called Shafer's model of FoD. The set of all subsets of $\Theta$ is called the powerset of $\Theta$ and it is denoted $2^{\Theta}$. A basic belief assignment (BBA) $m(\cdot)$ is defined as a mapping $m(\cdot): 2^{\Theta} \mapsto[0,1]$, verifying $m(\emptyset)=0$ and $\sum_{A \in 2^{\Theta}} m(A)=1$. In DSmT [2], one can abandon Shafer's model ${ }^{2}$ and refute the principle of the third excluded middle. Instead of defining the BBAs on the power set $2^{\Theta} \triangleq(\Theta, \cup)$, the BBAs are defined on the so-called hyper-power set (or Dedekind's lattice) denoted $D^{\Theta} \triangleq(\Theta, \cup, \cap)$ whose cardinalities follows Dedekind's numbers sequence [2], Vol.1. A (generalized) BBA is defined as the mapping $m(\cdot): D^{\Theta} \mapsto[0,1]$, verifying $m(\emptyset)=0$ and $\sum_{A \in D^{\Theta}} m(A)=1$. The DSmT framework encompasses DST framework because $2^{\Theta} \subset D^{\Theta}$. If $m(A)>0, A$ is called focal element of the BBA $m(\cdot)$. A BBA is called a Bayesian BBA if all of its focal elements are singletons and Shafer's model is assumed, otherwise it is called non-Bayesian. A full ignorance source is represented by the vacuous BBA $m_{v}(\Theta)=1$. In DST, the combination of several distinct SoE is done with the controversial Dempster's rule of combination which is the normalized version of conjunctive rule [1]. To palliate

\footnotetext{
${ }^{2}$ If Shafer's model doesn't fit with the problem.
}

the drawbacks of Dempster's rule, the Proportional Conflict Redistribution Rule \# 6 (PCR6) has been proposed in DSmT. Because we do not use these rules in this work, we do not need to present them in details here.

\section{FUSION OF EVIDENCES BASED ON RST}

\section{A. Construction of GS of TFE}

Consider a FoD $\Theta=\left\{\theta_{1}, \theta_{2}, \cdots, \theta_{n}\right\},(n \geq 2)$. We denote $X$ a specific TFE under concern. We denote $U=$ $\left\{u_{1}, u_{2}, \cdots, u_{i}\right\}$ a chosen subset of $D^{\Theta}$ corresponding to the set of focal elements of the BBA $m(\cdot)$ of the SoE. According to Pawlak's RST [7], any set $U$ can be partitioned from some chosen rules which help to determine if two elements of $U$ can be considered as equivalent or relevant, or not. For example, if the chosen rule is the belief mass, we will say that $u_{i} \in U$ and $u_{j} \in U$ are equivalent if and only if $m\left(u_{i}\right)=m\left(u_{j}\right)$. Using RST notations and formalism [7], the relation $I N D(R)$ defined by (1) is called a $R$-indiscernibility relation.

$$
I N D(R) \triangleq\left\{\left(u_{i}, u_{j}\right) \in U \times U \mid \forall r \in R, r\left(u_{i}\right)=r\left(u_{j}\right)\right\}
$$

If $\left(u_{i}, u_{j}\right) \in I N D(R)$, then $u_{i}$ and $u_{j}$ are indiscernible (or indistinguishable) from $R$. We denote $U(R) \triangleq\left\{\left[u_{i}\right]_{R} \mid u_{i} \in\right.$ $U\}$ the partition of $U$ based on $R$. $\left[u_{i}\right]_{R}$ denotes the equivalent class of $u_{i}$ with respect to $R$, i.e., $\left[u_{i}\right]_{R}=\left\{u_{j} \in U \mid r\left(u_{j}\right)=\right.$ $\left.r\left(u_{i}\right)\right\}$. The partition $U(R)$ is interpreted as a GS built from equivalence relation $R$. In fact, $R$ is a partitioning method which can discriminate elements of $U$ into separate subsets. With different equivalence relations, we can construct various meaningful partitions (GSs) of $U$.

Example 1: Consider a set $U=\left\{u_{1}, \ldots, u_{10}\right\}$ of focal elements of a normalized BBA $m(\cdot)$ whose values are

$$
\begin{array}{lll}
m\left(u_{1}\right)=0.0156 & m\left(u_{2}\right)=0.0874 & m\left(u_{3}\right)=0.0156 ; \\
m\left(u_{4}\right)=0.0874 & m\left(u_{5}\right)=0.0874 & m\left(u_{6}\right)=0.2354 \\
m\left(u_{7}\right)=0.2354 & m\left(u_{8}\right)=0.2354 & m\left(u_{9}\right)=0.0002 ; \\
m\left(u_{10}\right)=0.0002 & &
\end{array}
$$

If we consider the simple equivalence rule $R$ defined by

$$
R: u_{i} \equiv u_{j} \text { if and only if } m\left(u_{i}\right)=m\left(u_{j}\right)
$$

we see that $U$ can be partitioned as follows:

$$
U(R)=\left\{\left\{u_{1}, u_{3}\right\},\left\{u_{2}, u_{4}, u_{5}\right\},\left\{u_{6}, u_{7}, u_{8}\right\},\left\{u_{9}, u_{10}\right\}\right\}
$$

Of course, if a different rule is adopted, an other GS of $U$ is generated. In fusion problems, one has to consider several initial FoDs which are directly provided by the SoE. Each FoD associated with a source is related to the FoD corresponding to the scope of expertise of the SoE.

Example 2: Consider three distinct FoDs $\Theta_{1}=\left\{x_{1}, x_{2}, x_{3}\right\}$, $\Theta_{2}=\left\{y_{1}, y_{2}\right\}$ and $\Theta_{3}=\left\{z_{1}, z_{2}\right\}$ representing radar cross section (RCS) attributes, shape attributes and speed attributes, respectively. Suppose that one observes a target $T$ among a family of potential targets $\Theta_{T} \triangleq\left\{T_{1}, T_{2}, T_{3}, T_{4}, T_{5}, T_{6}\right\}$ and each target is characterized by its 3-tuple $\left(x_{i}, y_{j}, z_{k}\right)$. For example, $T_{1}=\left(x_{1}, y_{1}, z_{1}\right), T_{2}=\left(x_{1}, y_{2}, z_{1}\right), T_{3}=\left(x_{2}, y_{2}, z_{2}\right)$, 
$T_{4}=\left(x_{3}, y_{2}, z_{1}\right), T_{5}=\left(x_{3}, y_{1}, z_{1}\right)$, and $T_{6}=\left(x_{2}, y_{1}, z_{2}\right)$. Suppose that three smart sensors (i.e. sources of evidence) provide three reports expressed by the following BBAs

$$
\begin{aligned}
& m_{1}\left(x_{1}\right)=0.1, m_{1}\left(x_{3}\right)=0.2, m_{1}\left(x_{1} \cup x_{2}\right)=0.3, \\
& m_{1}\left(x_{1} \cup x_{2} \cup x_{3}\right)=0.4 \\
& m_{2}\left(y_{1}\right)=0.2, m_{2}\left(y_{2}\right)=0.3, m_{2}\left(y_{1} \cup y_{2}\right)=0.5 \\
& m_{3}\left(z_{1}\right)=0.8, m_{3}\left(z_{2}\right)=0, m_{3}\left(z_{1} \cup z_{2}\right)=0.2 .
\end{aligned}
$$

From these three SoE, one gets the three FoDs (i.e. sets of focal elements) $U_{1}, U_{2}$ and $U_{3}$

$$
\begin{aligned}
& U_{1}=\left\{x_{1}, x_{3}, x_{1} \cup x_{2}, x_{1} \cup x_{2} \cup x_{3}\right\} ; \\
& U_{2}=\left\{y_{1}, y_{2}, y_{1} \cup y_{2}\right\} ; \\
& U_{3}=\left\{z_{1}, z_{2}, z_{1} \cup z_{2}\right\} .
\end{aligned}
$$

These three sets of focal elements can be understood as three different GSs obtained by three independent experts using their prior knowledge in order to partition the set $U_{123} \triangleq U_{1} \times U_{2} \times U_{3}$.

In order to compute the global (fusioned) belief mass of any TFE $X$ from the available FoDs $U_{1}, U_{2}$ and $U_{3}$, we propose two works with two distinct domains called: the compatible domain and the incompatible domain of $X$, whose definitions are given below.

Definition 1 (Compatible domain of $X$ ): Consider $q \geq 2$ focal sets $U_{1}, U_{2}, \ldots, U_{q}$ provided by $q$ SoE. The compatible domain of a TFE $X=\left(x_{i_{1}}, \ldots, x_{i_{q}}\right)$, denoted $U_{1, \ldots, q}^{C}(X)$, corresponds to the set of all $q$-tuples of $U_{1} \times \ldots \times U_{q}$ and each componentwise intersection of q-tuple of $U_{1} \times U_{2} \times U_{q}$ with $X$ must be equal to the component of $X$ which means that the $q$ tuples of $U_{1} \times \ldots \times U_{q}$ is compatible with $X$. Mathematically, the compatible-domain of $X$ is defined by

$$
\begin{aligned}
& U_{1, \ldots, q}^{C}(X) \triangleq\left\{\left(u_{i_{1}}, \ldots, u_{i_{q}}\right) \in U_{1} \times \ldots \times U_{q}\right. \\
& \left.\mid \forall u_{i_{k}} \in U_{k}, u_{i_{k}} \cap x_{i_{k}}=x_{i_{k}}, x_{i_{k}} \in X, k=1, \ldots, q\right\} .
\end{aligned}
$$

For instance, taking back the example 2 and considering the TFE $T_{1}=\left(x_{1}, y_{1}, z_{1}\right)$, all 3-tuples that satisfy definition 1 are listed in Table I.

Interpreting the conflict as a situation which cannot occur (i.e. an impossible case), we define the incompatible domain of a given TFE $X$ as the set of all elements of the product space $U_{1} \times U_{2} \times \ldots \times U_{q}$ which have at least one component incompatible (or in conflict) with $X$. This yields to the following definition:

Definition 2 (Incompatible domain with $X$ ): Consider a TFE $X=\left(x_{i_{1}}, \ldots, x_{i_{q}}\right)$, and $q \geq 2$ focal sets $U_{1}, U_{2}, \ldots, U_{q}$ provided by $q$ SoE. The incompatible domain with $X$ (or conflict domain) denoted $U_{1, \ldots, q}^{I(X)}(\emptyset)$ corresponds to the set of all $q$-tuples of $U_{1} \times \ldots \times U_{q}$ having at least a componentwise empty intersection with $X$, that is

$$
\begin{aligned}
U_{1, \ldots, q}^{I(X)}(\emptyset) \triangleq\left\{\left(u_{i_{1}}, \ldots, u_{i_{q}}\right) \in U_{1} \times \ldots \times U_{q}\right. \\
\left.\mid \exists u_{i_{k}} \in U_{k}, u_{i_{k}} \cap x_{i_{k}}=\emptyset\right\} .
\end{aligned}
$$

Example 2 (revisited):

We show how to build these two domains for Example 2. As mentioned before the set of focal elements available from the three SoE are

$$
\begin{aligned}
& U_{1}=\left\{x_{1}, x_{3}, x_{1} \cup x_{2}, x_{1} \cup x_{2} \cup x_{3}\right\} \\
& U_{2}=\left\{y_{1}, y_{2}, y_{1} \cup y_{2}\right\} \\
& U_{3}=\left\{z_{1}, z_{2}, z_{1} \cup z_{2}\right\} .
\end{aligned}
$$

Because $U_{1}$ has 4 elements, $U_{2}$ has 3 elements and $U_{3}$ has 3 elements, the Cartesian product $U_{1} \times U_{2} \times U_{3}$ has $4 \times 3 \times 3=36$ elements. We suppose that the TFE under concern is $X=$ $T_{1}=\left(x_{1}, y_{1}, z_{1}\right)$. Based on the definitions 1 and 2 , the self domain and the conflict domain of $T_{1}$, are given as follows:

- Compatible domain of $T_{1}$ : All the elements of $U_{1,2,3}^{C}\left(T_{1}\right)$ are listed in Table I.

- Incompatible domain of $T_{1}$ : The set $U_{1,2,3}^{I\left(T_{1}\right)}(\emptyset)$ is the complement of $U_{1,2,3}^{C}\left(T_{1}\right)$ in $U_{1} \times U_{2} \times U_{3}$, that is $U_{1,2,3}^{I\left(T_{1}\right)}(\emptyset)=U_{1} \times U_{2} \times U_{3}-U_{1,2,3}^{C}\left(T_{1}\right)$, where the minus operator symbol corresponds to the set difference operator. $U_{1,2,3}^{I\left(T_{1}\right)}(\emptyset)$ has therefore $24=36-12$ elements (not listed here due to space limitation constraint).

The product set $U_{1} \times U_{2} \times U_{3}$ has been partitioned into two subsets, the compatible domain of $T_{1}$ including the twelve elements of $U_{1} \times U_{2} \times U_{3}$ compatible with $T_{1}$, and the incompatible domain of $T_{1}$ including the 24 elements incompatible with $T_{1}$.

Link with RST: From the standpoint of RST, we can define some equivalence rules based on definitions 1 and 2 , denoted $R=\left\{r_{1}=\right.$ rule based on definition $1, r_{2}=$ rule based on definition 2$\}$. Under a chosen rule $r \in R$, some particular elements $\left(u_{i_{1}}, \ldots, u_{i_{q}}\right)$ of $U_{1} \times \ldots \times U_{q}$ can be selected to characterize some special relationship with TFE (based on equivalence relation using definition 1 and conflict relation using definition 2). According to the principles of RST: $\mathrm{GSs}^{3}$ can be induced by various relations depending on the specificity of the problem, such as a neighborhood relation or a tolerance relation [7]. In the following example, we will show how a tolerance relation is used to solve the case where we have incomplete information of targets.

Dealing with incomplete information: In many practical problems, the available information can be incomplete. For example, it could happen that an object under interest has a (or more) missing (non observed) attribute. In such situation, this requires to deal with incomplete information with a special notation using the question mark symbol ? to represents the missing information. For instance, if the third attribute for the target $T_{1}$ of example 2 is missing (unknown), we will denote it as $\left(x_{1}, y_{1}, ?\right)$ and question mark symbol ? represents the third missing attribute. Because of the possibility of missing attribute, the definition 1 needs to be revised as follows ${ }^{4}$ :

\footnotetext{
${ }^{3}$ In this paper, $U_{1, \ldots, q}^{C}(X)$ and $U_{1, \ldots, q}^{I(X)}(\emptyset)$ are regarded as a variety of GSs in RST.

${ }^{4}$ Modification of definition 2 is similar.
} 
TABLE I: Elements of $U_{1,2,3}^{C}\left(T_{1}\right)$ for Example 2.

\begin{tabular}{|c|c|c|c|}
\hline$\left(x_{1}, y_{1}, z_{1}\right)$ & $\left(x_{1}, y_{1} \cup y_{2}, z_{1}\right)$ & $\left(x_{1}, y_{1}, z_{1} \cup z_{2}\right)$ & $\left(x_{1}, y_{1} \cup y_{2}, z_{1} \cup z_{2}\right)$ \\
\hline$\left(x_{1} \cup x_{2}, y_{1}, z_{1}\right)$ & $\left(x_{1} \cup x_{2}, y_{1} \cup y_{2}, z_{1}\right)$ & $\left(x_{1} \cup x_{2}, y_{1}, z_{1} \cup z_{2}\right)$ & $\left(x_{1} \cup x_{2}, y_{1} \cup y_{2}, z_{1} \cup z_{2}\right)$ \\
\hline$\left(x_{1} \cup x_{2} \cup x_{3}, y_{1}, z_{1}\right)$ & $\left(x_{1} \cup x_{2} \cup x_{3}, y_{1} \cup y_{2}, z_{1}\right)$ & $\left(x_{1} \cup x_{2} \cup x_{3}, y_{1}, z_{1} \cup z_{2}\right)$ & $\left(x_{1} \cup x_{2} \cup x_{3}, y_{1} \cup y_{2}, z_{1} \cup z_{2}\right)$ \\
\hline
\end{tabular}

Definition 3 (Compatible domain with incomplete information): Consider $q \geq 2$ focal sets $U_{1}, U_{2}, \ldots, U_{q}$ provided by $q$ SoE. The compatible domain of a TFE $X$ with missing attributes, denoted $U_{1, \ldots, q}^{\prime} C(X)$, corresponds to the set of all $q$ tuples of $U_{1} \times \ldots \times U_{q}$ and each componentwise intersection of q-tuple of $U_{1} \times U_{2} \times U_{q}$ with $X$ must be equal to the component of $X$, that is

$$
\begin{aligned}
& U_{1, \ldots, q}^{\prime C}(X) \triangleq\left\{\left(u_{i_{1}}, \ldots, u_{i_{q}}\right) \in U_{1} \times \ldots \times U_{q}\right. \\
& \left.\mid \forall u_{i_{k}} \in U_{k}, u_{i_{k}} \cap x_{i_{k}}=x_{i_{k}} \text { or } u_{i_{k}} \rightarrow ?, k=1, \ldots, q\right\} .
\end{aligned}
$$

Here, $u_{i_{k}} \rightarrow$ ? means that for all $u_{i_{k}}$ in $U_{k}$ replacing the missing attribute in $k$-th dimension of $X$, respectively. And $u_{i_{k}} \rightarrow$ ? actually means $u_{i_{k}} \cap ? \equiv u_{i_{k}}, k=1, \ldots, q$.

Considering back the example 2 , if $X=\left(x_{1}, y_{1}, ?\right)$, we will get $U_{1, \ldots, 3}^{\prime}(X)$ as follows:

$$
\begin{aligned}
& U_{1, \ldots, 3}^{\prime C}(X)=\left\{\left(x_{1}, y_{1}, z_{1}\right),\left(x_{1}, y_{1} \cup y_{2}, z_{1}\right),\right. \\
& \left(x_{1}, y_{1}, z_{1} \cup z_{2}\right),\left(x_{1}, y_{1} \cup y_{2}, z_{1} \cup z_{2}\right),\left(x_{1} \cup x_{2}, y_{1}, z_{1}\right), \\
& \left(x_{1} \cup x_{2}, y_{1} \cup y_{2}, z_{1}\right),\left(x_{1} \cup x_{2}, y_{1}, z_{1} \cup z_{2}\right), \\
& \left(x_{1} \cup x_{2}, y_{1} \cup y_{2}, z_{1} \cup z_{2}\right),\left(x_{1} \cup x_{2} \cup x_{3}, y_{1}, z_{1}\right), \\
& \left(x_{1} \cup x_{2} \cup x_{3}, y_{1} \cup y_{2}, z_{1}\right),\left(x_{1} \cup x_{2} \cup x_{3}, y_{1}, z_{1} \cup z_{2}\right), \\
& \left(x_{1} \cup x_{2} \cup x_{3}, y_{1} \cup y_{2}, z_{1} \cup z_{2}\right),\left(x_{1}, y_{1}, z_{2}\right), \\
& \left(x_{1} \cup x_{2}, y_{1}, z_{2}\right),\left(x_{1} \cup x_{2} \cup x_{3}, y_{1}, z_{2}\right), \\
& \left(x_{1}, y_{1} \cup y_{2}, z_{2}\right),\left(x_{1} \cup x_{2}, y_{1} \cup y_{2}, z_{2}\right), \\
& \left.\left(x_{1} \cup x_{2} \cup x_{3}, y_{1} \cup y_{2}, z_{2}\right)\right\}
\end{aligned}
$$

The elements of $U_{1, \ldots, 3}^{\prime C}(X)$ are the triplets of $U_{1} \times U_{2} \times U_{3}$ compatible with the two first components of $X$ for which the missing attribute of $X$ is replaced either by $z_{1}, z_{2}$, or $z_{1} \cup z_{2}$.

The definition 3 can also be regarded as a kind of equivalence relation ${ }^{5}$ which is applied to find the relevant sets of TFE in $U_{1} \times \ldots \times U_{q}$.

The main contribution of this paper is to propose some rules to handle the different domains of TFE based on the specificity of the problem. Based on these rules, we can identify the compatible-domain and the incompatible domain for a chosen TFE, and then define belief allocation strategies and combination in order to get a final quantitative result as proposed in the sequel.

\section{B. Calculation of the belief mass of TFE}

Once the compatible domain and the incompatible domain of TFE are identified, we use the elements in these domains

\footnotetext{
${ }^{5}$ This equivalence relation is also called tolerance relation in RST.
}

to calculate the belief mass of a chosen TFE. In fact, in RST, the granular space obtained by each partitioning rule will be used to approximate the target concepts, namely the upper approximation set and the lower approximation set, as well as the positive, negative and uncertainty domains about the target concept. Such results of approximation are to some extent qualitative expressions. Once equivalent sets of TFE in each domain are found, we calculate the belief mass of TFE in each domain space in order to get a quantitative result. In a certain sense, rough set solves the qualitative solution of target concept and the multi-granular rough set fusion is a kind of qualitative fusion method, whereas the evidence fusion provides a quantitative fusion solution. Although the two approaches are focused on different aspects, from a fusion point of view, the rough set approach can be seen as complementary to the fusion rules of belief functions. In what follows, we propose a method to combine BBAs to get the belief mass of TFE based on the construction of own domain and conflict domain.

\section{Fusion based on partially uniform redistribution rule}

Consider $q \geq 2$ BBAs $m_{1}(\cdot), \ldots, m_{q}(\cdot)$ with $q$ sets of focal elements $U_{1}, \ldots, U_{q}$, and a subset $X$ of $U_{1} \times U_{2} \times \cdots \times U_{q}$. The (conjunctive) fusion for the TFE $X$ is calculated by

$$
m_{1, \ldots, q}^{C}(X)=\sum_{\left(u_{i_{1}}, \ldots, u_{i_{q}}\right) \in U_{1, \ldots, q}^{C}(X)} m_{1}\left(u_{i_{1}}\right) \cdot \ldots \cdot m_{q}\left(u_{i_{q}}\right) .
$$

To reduce the complexity of the fusion combination rule, we propose to average the conflicting masses allocated to elements of $U_{1 \ldots q}^{I(X)}(\emptyset)$ and to distribute it back to $X$, which is expressed as

$$
\frac{m_{1, \ldots, q}(X)}{\left|U_{1 \ldots q}^{I(X)}(\emptyset)\right|} \sum_{\left(u_{i_{1}}, \ldots, u_{i_{q}}\right) \in U_{1 \ldots q}^{I(X)}(\emptyset)}^{C} m_{1}\left(u_{i}\right) \times \ldots \times m_{q}\left(u_{i_{q}}\right) .
$$

where $\left|U_{1 \ldots q}^{I(X)}(\emptyset)\right|$ is the cardinality of $U_{1 \ldots q}^{I(X)}(\emptyset)$.

With such basic redistribution principle, after having computed the BBA $m_{1 \ldots q}(X)$ of all possible TFE $X$, we don't get a normalized BBA. That is why a normalization step must be applied to get final fusion result, 
Example 2 (revisited): Let's consider $X=T_{1}=\left(x_{1}, y_{1}, z_{1}\right)$, then based on Table I one has

$$
\begin{aligned}
& U_{1,2,3}^{C}\left(T_{1}\right)=\left\{\left(x_{1}, y_{1}, z_{1}\right),\left(x_{1}, y_{1} \cup y_{2}, z_{1}\right),\right. \\
& \left(x_{1}, y_{1}, z_{1} \cup z_{2}\right),\left(x_{1}, y_{1} \cup y_{2}, z_{1} \cup z_{2}\right),\left(x_{1} \cup x_{2}, y_{1}, z_{1}\right), \\
& \left(x_{1} \cup x_{2}, y_{1} \cup y_{2}, z_{1}\right),\left(x_{1} \cup x_{2}, y_{1}, z_{1} \cup z_{2}\right), \\
& \left(x_{1} \cup x_{2}, y_{1} \cup y_{2}, z_{1} \cup z_{2}\right),\left(x_{1} \cup x_{2} \cup x_{3}, y_{1}, z_{1}\right), \\
& \left(x_{1} \cup x_{2} \cup x_{3}, y_{1} \cup y_{2}, z_{1}\right),\left(x_{1} \cup x_{2} \cup x_{3}, y_{1}, z_{1} \cup z_{2}\right), \\
& \left.\left(x_{1} \cup x_{2} \cup x_{3}, y_{1} \cup y_{2}, z_{1} \cup z_{2}\right)\right\} .
\end{aligned}
$$

The (conjunctive) mass of belief of $T_{1}$ is given by

$$
\begin{aligned}
m_{1, \ldots, 3}^{C}\left(T_{1}\right)= & \sum_{\left(u_{i_{1}}, u_{i_{2}}, u_{i_{3}}\right) \in U_{1,2,3}^{C}(X)} m_{1}\left(u_{i_{1}}\right) m_{2}\left(u_{i_{2}}\right) m_{3}\left(u_{i_{3}}\right) \\
= & m_{1}\left(x_{1}\right) m_{2}\left(y_{1}\right) m_{3}\left(z_{1}\right) \\
& +m_{1}\left(x_{1}\right) m_{2}\left(y_{1}\right) m_{3}\left(z_{1} \cup z_{2}\right) \\
& +m_{1}\left(x_{1} \cup x_{2}\right) m_{2}\left(y_{1}\right) m_{3}\left(z_{1}\right) \\
& +m_{1}\left(x_{1} \cup x_{2}\right) m_{2}\left(y_{1}\right) m_{3}\left(z_{1} \cup z_{2}\right) \\
& +m_{1}\left(x_{1} \cup x_{2} \cup x_{3}\right) m_{2}\left(y_{1}\right) m_{3}\left(z_{1}\right) \\
& +m_{1}\left(x_{1} \cup x_{2} \cup x_{3}\right) m_{2}\left(y_{1}\right) m_{3}\left(z_{1} \cup z_{2}\right) \\
& +m_{1}\left(x_{1}\right) m_{2}\left(y_{1} \cup y_{2}\right) m_{3}\left(z_{1}\right) \\
& +m_{1}\left(x_{1}\right) m_{2}\left(y_{1} \cup y_{2}\right) m_{3}\left(z_{1} \cup z_{2}\right) \\
& +m_{1}\left(x_{1} \cup x_{2}\right) m_{2}\left(y_{1} \cup y_{2}\right) m_{3}\left(z_{1}\right) \\
& +m_{1}\left(x_{1} \cup x_{2}\right) m_{2}\left(y_{1} \cup y_{2}\right) m_{3}\left(z_{1} \cup z_{2}\right) \\
& +m_{1}\left(x_{1} \cup x_{2} \cup x_{3}\right) m_{2}\left(y_{1} \cup y_{2}\right) m_{3}\left(z_{1}\right) \\
& +m_{1}\left(x_{1} \cup x_{2} \cup x_{3}\right) m_{2}\left(y_{1} \cup y_{2}\right) m_{3}\left(z_{1}\right) \\
= & 0.56 .
\end{aligned}
$$

The incompatible domain $U_{1 \ldots q}^{I(X)}(\emptyset)$ has 24 elements. They correspond altogether to a conflicting belief mass value given by the total mass value (equal to one) minus the mass of belief of the 12 q-tuples compatible with $T_{1}$. That is

$$
m_{1 \ldots 3}^{I\left(T_{1}\right)}(\emptyset)=1.0-0.56=0.44
$$

Therefore the average conflicting mass value is. $\frac{1}{\left|U_{1 \ldots q}^{I(X)}(\emptyset)\right|} m_{1 \ldots 3}^{I\left(T_{1}\right)}(\emptyset)=\frac{1}{24} \cdot 0.44 \approx 0.0183$.

Thus the total belief mass committed to $T_{1}$ (before normalization) will be

$$
\begin{aligned}
m_{1, \ldots, 3}\left(T_{1}\right) & =m_{1, \ldots, 3}^{C}\left(T_{1}\right)+\frac{1}{\left|U_{1 \ldots q}^{I(X)}(\emptyset)\right|} m_{1 \ldots 3}^{I\left(T_{1}\right)}(\emptyset) \\
& =0.56+\frac{1}{24} \cdot 0.44 \approx 0.5783 .
\end{aligned}
$$

We apply the same principle of derivation for all targets of $\Theta_{T}$. After the normalization step, we finally get

$$
\begin{array}{ll}
m_{123}\left(T_{1}\right) \approx 0.2274 & m_{123}\left(T_{2}\right) \approx 0.2485 \\
m_{123}\left(T_{3}\right) \approx 0.0558 & m_{123}\left(T_{4}\right) \approx 0.2174 \\
m_{123}\left(T_{5}\right) \approx 0.1998 & m_{123}\left(T_{6}\right) \approx 0.0511 .
\end{array}
$$

Example 3 (revisited): We consider the following TFE $X=$ $T^{*}=\left(x_{1}, y_{1}, ?\right)$ with a missing attribute (the third component is missing). Then, one has

$$
\begin{aligned}
& U_{1,2,3}^{\prime C}\left(T^{*}\right)=\left\{\left(x_{1}, y_{1}, z_{1}\right),\left(x_{1}, y_{1} \cup y_{2}, z_{1}\right),\right. \\
& \left(x_{1}, y_{1}, z_{1} \cup z_{2}\right),\left(x_{1}, y_{1} \cup y_{2}, z_{1} \cup z_{2}\right),\left(x_{1} \cup x_{2}, y_{1}, z_{1}\right), \\
& \left(x_{1} \cup x_{2}, y_{1} \cup y_{2}, z_{1}\right),\left(x_{1} \cup x_{2}, y_{1}, z_{1} \cup z_{2}\right), \\
& \left(x_{1} \cup x_{2}, y_{1} \cup y_{2}, z_{1} \cup z_{2}\right),\left(x_{1} \cup x_{2} \cup x_{3}, y_{1}, z_{1}\right), \\
& \left(x_{1} \cup x_{2} \cup x_{3}, y_{1} \cup y_{2}, z_{1}\right),\left(x_{1} \cup x_{2} \cup x_{3}, y_{1}, z_{1} \cup z_{2}\right), \\
& \left(x_{1} \cup x_{2} \cup x_{3}, y_{1} \cup y_{2}, z_{1} \cup z_{2}\right),\left(x_{1}, y_{1}, z_{2}\right), \\
& \left(x_{1} \cup x_{2}, y_{1}, z_{2}\right),\left(x_{1} \cup x_{2} \cup x_{3}, y_{1}, z_{2}\right), \\
& \left(x_{1}, y_{1} \cup y_{2}, z_{2}\right),\left(x_{1} \cup x_{2}, y_{1} \cup y_{2}, z_{2}\right), \\
& \left.\left(x_{1} \cup x_{2} \cup x_{3}, y_{1} \cup y_{2}, z_{2}\right)\right\}
\end{aligned}
$$

Compared to the $U_{1,2,3}^{C}\left(T_{1}\right), U_{1,2,3}^{\prime}\left(T^{*}\right)$ contains six extra elements. The (conjunctive) mass of belief of $T^{*}$ is given by

$$
\begin{aligned}
m_{1,2,3}^{\prime C}\left(T^{*}\right)= & 0.56+m_{1}\left(x_{1}\right) m_{2}\left(y_{1}\right) m_{3}\left(z_{2}\right) \\
& +m_{1}\left(x_{1} \cup x_{2}\right) m_{2}\left(y_{1}\right) m_{3}\left(z_{2}\right) \\
& +m_{1}\left(x_{1} \cup x_{2} \cup x_{3}\right) m_{2}\left(y_{1}\right) m_{3}\left(z_{2}\right) \\
& +m_{1}\left(x_{1}\right) m_{2}\left(y_{1} \cup y_{2}\right) m_{3}\left(z_{2}\right) \\
& +m_{1}\left(x_{1} \cup x_{2}\right) m_{2}\left(y_{1} \cup y_{2}\right) m_{3}\left(z_{2}\right) \\
& +m_{1}\left(x_{1} \cup x_{2} \cup x_{3}\right) m_{2}\left(y_{1} \cup y_{2}\right) m_{3}\left(z_{2}\right) \\
= & 0.56+0=0.56 .
\end{aligned}
$$

The conflicting mass of $T^{*}$ is given by the total mass value (equal to one) minus the mass of belief of all the 18 triplets compatible with $T^{*}$, that is

$$
m_{1,2,3}^{\prime I\left(T^{*}\right)}(\emptyset)=1-0.56=0.44 .
$$

Because $U_{1,2,3}^{I\left(T^{*}\right)}(\emptyset)$ has $36-18=18$ elements, one gets

$$
\begin{aligned}
m_{1,2,3}^{\prime}\left(T^{*}\right)= & m_{1,2,3}^{\prime C}\left(T^{*}\right)+\frac{1}{18} m_{1,2,3}^{\prime I\left(T^{*}\right)}(\emptyset) \\
& =0.56+\frac{1}{18} \cdot 0.44 \approx 0.5844 .
\end{aligned}
$$

\section{Summary OF FUSION BASED ON RST}

Figure 1 shows the whole diagram of our proposed combination method and the most important steps are the construction of GSs and calculation of belief mass of TFE. In the following example, we choose conflict allocation in new combination method to fuse sources with distinct FoDs.

\section{Application Example}

Here, we will use the target identification example available in the literature [8] to illustrate our new method for combining SoE with distinct FoDs. This example is about an air surveillance system, which determines the targets' ID by combining the outputs of multiple sensors. Suppose that the FoD for the target allegiance is $\Theta_{A}$, and the FoD for the target classes is $\Theta_{B}^{r e f}$. In our example, one has $\Theta_{A}=\{f, n, s, h\}$, where $f \triangleq$ friend, $n \triangleq$ neutral, $s \triangleq$ suspect, $h \triangleq$ hostile and $\Theta_{B}=\left\{B_{1}, B_{2}, B_{3}, B_{4}\right\}$ : 
TABLE II: Available Sensor Reports.

\begin{tabular}{|l|c|c|c|}
\hline Report ID & \multicolumn{3}{|c|}{ Corresponding evidence BoE } \\
\hline Report $^{(1)}$ & $m_{\Theta_{B}}^{(1)}\left(B_{2}\right)=0.7$ & $m_{\Theta_{B}}^{(1)}\left(B_{2}, B_{3}\right)=0.2$ & $m_{\Theta_{B}}^{(1)}\left(\Theta_{B}\right)=0.1$ \\
\hline $\operatorname{Report}^{(2)}$ & $m_{\Theta_{B}^{\text {ref }}}^{(2)}\left(b_{22}\right)=0.6$ & $m_{\Theta_{B}^{\text {ref }}}^{(2)}\left(b_{23}\right)=0.3$ & $m_{\Theta_{B}^{(2)}}^{(2)}\left(\Theta_{B}^{\text {ref }}\right)=0.1$ \\
\hline $\operatorname{Report}^{(3)}$ & $m_{\Theta_{A}}^{(3)}(s)=0.6$ & $m_{\Theta_{A}}^{(3)}\left(\Theta_{A}\right)=0.4$ & - \\
\hline $\operatorname{Report}^{(4)}$ & $m_{\Theta_{A}}^{(3)}(f)=0.9$ & $m_{\Theta_{A}}^{(3)}\left(\Theta_{A}\right)=0.1$ & - \\
\hline
\end{tabular}

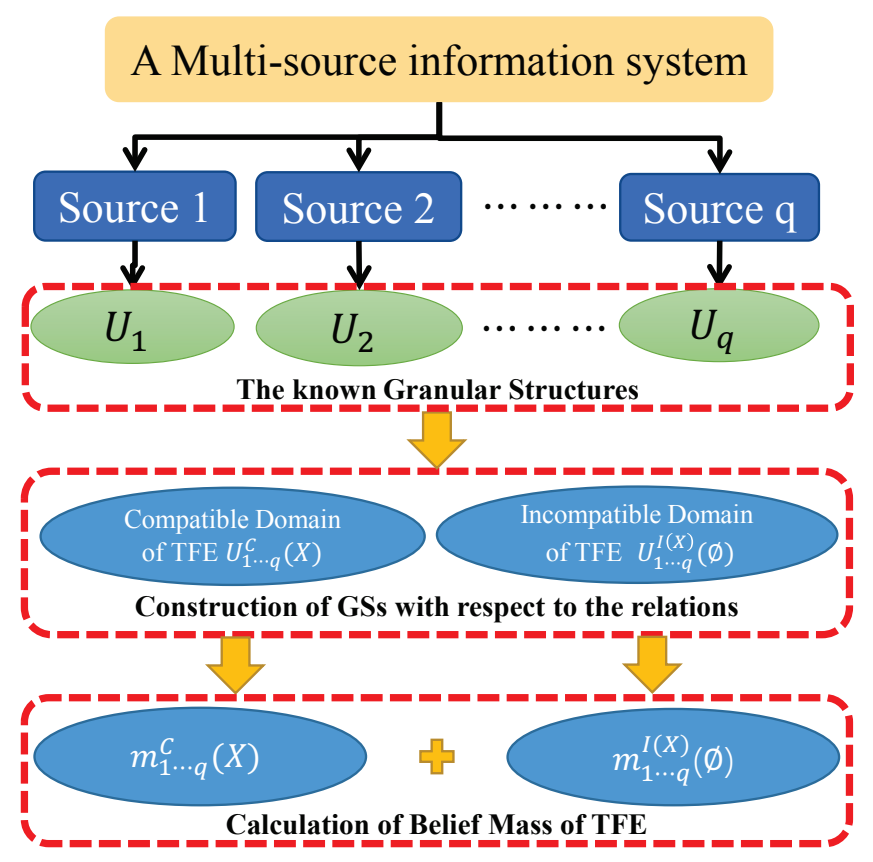

Fig. 1: Diagram of the proposed combination method.

$$
\begin{aligned}
& B_{1}=\text { Commerical Planes } \\
& B_{2}=\text { Fighter Planes } \\
& B_{3}=\text { Bombers } \\
& B_{4}=\text { Military Transport Planes. }
\end{aligned}
$$

Each basic target class consists of several platform types:

$$
\begin{aligned}
& B_{1}=\left\{b_{11}, b_{12}\right\} ;\left(e . g ., b_{11} \text { could be Airbus }-320\right) \\
& B_{2}=\left\{b_{21}, b_{22}, b_{23}, b_{24}, b_{25}\right\} \\
& B_{3}=\left\{b_{31}, b_{32}, b_{33}\right\} \\
& B_{4}=\left\{b_{41}, b_{42}\right\}\left(\text { e.g., } b_{41} \text { could be an } A 380 M\right) .
\end{aligned}
$$

Hence, the FoD $\Theta_{B}^{r e f}=B_{1} \cup B_{2} \cup B_{3} \cup B_{4}$ is the refinement of $\Theta_{B}$. Target ID belongs to the FoD $\Theta=\Theta_{A} \times \Theta_{B}^{r e f}$, for example, Target ID is $s \times b_{41}$, which means suspect an225 Mriya or $h \times b_{11}$ means hostile Airbus-320. The four sensor reports are shown in Table II, and fusion center combines all the sensor reports and generates a BBA for decisionmaking. Suppose that the pairs $\left\{\right.$ Report $^{(1)}$, Report $\left.^{(2)}\right\}$ and $\left\{\right.$ Report $^{(3)}$, Report $\left.^{(4)}\right\}$ are fused separately. The resulting BBAs are vacuously extended and fused by the new combination method in this paper so as to obtain the fused mass $m_{\Theta}(\cdot)$ as

$$
m_{\Theta}(\cdot)=\left(m_{\Theta_{B}}^{(1)}(\cdot) \oplus m_{\Theta_{B}^{r e f}}^{(2)}(\cdot)\right) \oplus\left(m_{\Theta_{A}}^{(3)}(\cdot) \oplus m_{\Theta_{A}}^{(4)}(\cdot)\right) .
$$

Based on the original data given by four sensor reports in Table II, we can obtain four initial GSs:

$$
\begin{aligned}
& U_{1}=\left\{B_{2},\left\{B_{2}, B_{3}\right\}, \Theta_{B}\right\} ; \\
& U_{2}=\left\{b_{22}, b_{23}, \Theta_{B}^{r e f}\right\} ; \\
& U_{3}=\left\{s, \Theta_{A}\right\} ; \\
& U_{4}=\left\{f, \Theta_{A}\right\} ;
\end{aligned}
$$

Based on definitions of self-domain, conflict-domain, we can obtain some new and interesting results which are shown in Table III, IV, V: In Table III, since focal elements in $U_{1}$ and $U_{2}$ exist inclusion relations shown in Figure 2, for example, $\left\{B_{2}, B_{3}\right\} \in \Theta_{B}, b_{22} \in B_{2}, b_{23} \in B_{2}$, thus, the intersections between each element in $U_{1}$ and $U_{2}$ are not $\emptyset$, and according to definitions 3 , the conflict domain is empty set, and the elements of its self domain are shown in Table III. Furthermore, according to definition 4, we can calculate the belief mass of $B_{2}: m_{\Theta_{B}}^{(1)}\left(B_{2}\right) \times m_{\Theta_{B}^{r e f}}^{(2)}\left(\Theta_{B}^{r e f}\right)=$ $0.7 \times 0.1=0.07$. Similarly, we can also calculate belief masses of $\left\{B_{2}, B_{3}\right\}, \Theta_{B}, b_{22}, b_{23}, \Theta_{B}^{r e f}$; Similarly, in Table IV, the conflict between $U_{3}$ and $U_{4}$ is caused by $s$ and $f$, thus, conflict domain includes these two elements. Then, based on Eq.(5) and Eq.(6), we can get the corresponding masses of $s, f$ and $A$, which are given in Table IV; Table $\mathrm{V}$ shows that the fused results based on the sensor reports above. Due to target ID belongs to $\Theta_{A} \times \Theta_{B}^{r e f}$ and taking into account the focal elements involved in Table II, the FoD $\Theta_{A} \times \Theta_{B}^{r e f}$ is reduced to $\Theta_{A^{\prime}} \times \Theta_{B^{\prime}}: \Theta_{A^{\prime}}=\left\{s, f, \Theta_{A}\right\}$ and $\Theta_{B^{\prime}}=\left\{b_{22}, b_{23}, B_{2},\left\{B_{2}, B_{3}\right\}, \Theta_{B}^{r e f}\right\}$. Then, in Table $\mathrm{V}$, we can get the self domains and conflict domains of all TFEs. Finally, we can get the belief masses of all elements in $\Theta_{A^{\prime}} \times \Theta_{B^{\prime}}$. Based on the maximum of belief mass principle, they appear to favor a target ID of $f \times b_{22}$ (i.e., a friendly $b_{22}$ ), which is consistent with the conclusions in [8] and [9].

Remark 3: This example shows two advantages of our new combination method in this paper: (1) we do not need to expand FoDs of four sensor reports to ensure that they are under the unique FoD. For example, if we use the classical 
TABLE III: Domains and belief masses of TFEs in $\left\{\right.$ Report $^{(1)}$, Report $\left.^{(2)}\right\}$.

\begin{tabular}{|c|c|c|c|}
\hline \multirow{2}{*}{ Target $X$} & \multicolumn{2}{|c|}{ Domain } & Mass of $\Theta_{B^{\prime}}$ \\
\cline { 2 - 3 } & $\begin{array}{c}\text { Compatible } \\
\text { domain }\end{array}$ & $\begin{array}{c}\text { Incompatible } \\
\text { Domain }\end{array}$ & \\
\hline$X=B_{2}$ & $\left\{\left(B_{2}, \Theta_{B}^{r e f}\right)\right\}$ & $\{\emptyset\}$ & 0.07 \\
\hline$X=\left\{B_{2}, B_{3}\right\}$ & $\left\{\left(B_{2} B_{3}, \Theta_{B}^{r e f}\right)\right\}$ & $\{\emptyset\}$ & 0.02 \\
\hline & $\begin{array}{c}\left\{\left(B_{2}, b_{22}\right),\right. \\
\left(B_{2} B_{3}, b_{22}\right), \\
\left.\left(\Theta_{B}, b_{22}\right)\right\}\end{array}$ & $\{\emptyset\}$ & 0.60 \\
\hline$X=b_{22}$ & $\begin{array}{c}\left\{\left(B_{2}, b_{23}\right),\right. \\
\left(B_{2} B_{3}, b_{23}\right), \\
\left.\left(\Theta_{B}, b_{23}\right)\right\}\end{array}$ & $\{\emptyset\}$ & 0.30 \\
\hline$X=b_{23}$ & $\left\{\left(\Theta_{B}, \Theta_{B}^{r e f}\right)\right\}$ & $\{\emptyset\}$ & 0.01 \\
\hline
\end{tabular}

TABLE IV: Domains and belief masses of TFEs in $\left\{\right.$ Report $^{(3)}$, Report $\left.^{(4)}\right\}$.

\begin{tabular}{|c|c|c|c|}
\hline \multirow{2}{*}{ Target $X$} & \multicolumn{2}{|c|}{ Domain } & Mass of $\Theta_{A^{\prime}}$ \\
\cline { 2 - 3 } & $\begin{array}{c}\text { Compatible } \\
\text { domain }\end{array}$ & $\begin{array}{c}\text { Incompatible } \\
\text { Domain }\end{array}$ & \\
\hline$X=s$ & $\left\{\left(s, \Theta_{A}^{(4)}\right)\right\}$ & $\{(s, f)\}$ & 0.24 \\
\hline$X=\Theta_{A}$ & $\left\{\left(\Theta_{A}^{(3)}, \Theta_{A}^{(4)}\right)\right\}$ & $\{\emptyset\}$ & 0.22 \\
\hline$X=f$ & $\left\{\left(f, \Theta_{A}^{(3)}\right)\right\}$ & $\{(s, f)\}$ & 0.54 \\
\hline
\end{tabular}

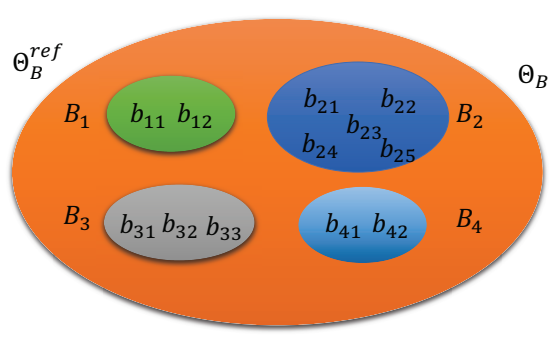

(a)

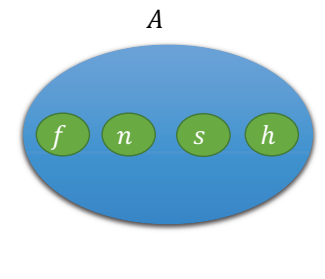

(b)
Fig. 2: Elements in $U_{1}, U_{2}$ (a) and $U_{3}, U_{4}$ (b).

combination method, first, we need to construct a common FoD $\Theta_{\text {expand }}=\left\{s, f, A, b_{22}, b_{23}, \Theta_{B}^{r e f}, B_{2},\left(B_{2}, B_{3}\right), \Theta_{B}\right\}$, and then we update the BBAs by assigning zero masses to the elements that are not in their specific FoDs. This expanded way will obviously increase the computational complexity; (2) On the other hand, the new combination method can effectively get the belief mass of the special single target (for example in this case, hostile Airbus-320) when there are no need to pay more attentions to other potential targets. However, all belief masses of targets will be calculated in a single calculation step in traditional combination ways.

\section{CONCLUSION}

In this paper we did propose a new combination method to solve the fusion problem of sources with distinct FoDs.
Specifically, the new fusion process is divided into two steps: 1) according to GSs provided by original sources, we construct the compatible domain and the incompatible domain of TFE based on several rules; and 2) we calculate the belief mass of TFE with very basic belief allocation strategy. Experiments show that the approach presented in this paper can provide a solution to the fusion of sources with distinct FoDs compatible with more sophisticate methods. In future works, we will continue to explore more rules to get better relevant sets of TFE and to improve the conflict redistribution strategies in order to combine more efficiently (hopefully) different sources defined on distinct FoDs.

\section{ACKNOWLEDGMENT}

This work was supported in part by the National Natural Science Foundation of China under Grant 61573097 and 91748106, in part by Key Laboratory of Integrated Automation of Process Industry (PAL-N201704), the Fundamental Research Funds for the Central Universities (3208008401), the Qing Lan Project and Six Major Top-talent Plan, and in part by the Priority Academic Program Development of Jiangsu Higher Education Institutions. The authors thank the reviewers and editors for giving valuable comments, which are very helpful for improving this manuscript.

\section{REFERENCES}

[1] G. Shafer, A mathematical theory of evidence. Princeton university press, 1976, vol. 42.

[2] F. Smarandache and J. Dezert, "Advances and applications of DSmT for information fusion," vol. 1-4, 2004-2015, https://www.onera.fr/staff/jeandezert/references.

[3] F. Janez and A. Appriou, "Theory of evidence and non-exhaustive frames of discernment: Plausibilities correction methods," International journal of approximate reasoning, vol. 18, no. 1-2, pp. 1-19, 1998.

[4] E. Kulasekere, K. Premaratne, D. A. Dewasurendra, M.-L. Shyu, and P. H. Bauer, "Conditioning and updating evidence," International Journal of Approximate Reasoning, vol. 36, no. 1, pp. 75-108, 2004.

[5] K. Premaratne, D. A. Dewasurendra, and P. H. Bauer, "Evidence combination in an environment with heterogeneous sources," IEEE Transactions on Systems, Man, and Cybernetics-Part A: Systems and Humans, vol. 37, no. 3, pp. 298-309, 2007.

[6] T. Wickramarathne, K. Premaratne, M. N. Murthi, M. Scheutz, S. Kübler, and M. Pravia, "Belief theoretic methods for soft and hard data fusion," in Acoustics, Speech and Signal Processing (ICASSP), 2011 IEEE International Conference on. IEEE, 2011, pp. 2388-2391.

[7] Z. Pawlak, Rough sets: Theoretical aspects of reasoning about data. Springer Science \& Business Media, 2012, vol. 9.

[8] B. Ristic and P. Smets, "Target identification using belief functions and implication rules," IEEE transactions on Aerospace and Electronic Systems, vol. 41, no. 3, pp. 1097-1103, 2005.

[9] T. L. Wickramarathne, "An analytical framework for soft and hard data fusion: A Dempster-Shafer belief theoretic approach," Ph.D. dissertation, University of Miami, 2012. 
TABLE V: Fused BoE generated from four sensor reports.

\begin{tabular}{|c|c|c|c|}
\hline \multirow[t]{2}{*}{ Target $X$} & \multicolumn{2}{|l|}{ Domain } & \multirow[t]{2}{*}{ Belief Mass } \\
\hline & Compatible domain & Incompatible Domain & \\
\hline$s \times b_{22}$ & $\begin{array}{c}\left\{\left(s, b_{22}\right),\left(s, B_{2}\right),\left(s, B_{2} B_{3}\right),\right. \\
\left(s, \Theta_{B}^{r e f}\right),\left(\Theta_{A}, b_{22}\right),\left(\Theta_{A}, B_{2}\right), \\
\left.\left(\Theta_{A}, B_{2} B_{3}\right),\left(\Theta_{A}, \Theta_{B}^{r e f}\right)\right\}\end{array}$ & $\begin{array}{c}\left\{\left(s, b_{23}\right),\left(\Theta_{A}, b_{23}\right),\left(f, b_{22}\right),\right. \\
\left(f, b_{23}\right),\left(f, B_{2}\right),\left(f, B_{2} B_{3}\right), \\
\left.\left(f, \Theta_{B}^{r e f}\right)\right\}\end{array}$ & 0.1601 \\
\hline$s \times b_{23}$ & $\begin{array}{c}\left\{\left(s, b_{23}\right),\left(s, B_{2}\right),\left(s, B_{2} B_{3}\right),\right. \\
\left(s, \Theta_{B}^{r e f}\right),\left(\Theta_{A}, b_{23}\right),\left(\Theta_{A}, B_{2}\right), \\
\left.\left(\Theta_{A}, B_{2} B_{3}\right),\left(\Theta_{A}, \Theta_{B}^{r e f}\right)\right\}\end{array}$ & $\begin{array}{c}\left\{\left(s, b_{22}\right),\left(\Theta_{A}, b_{22}\right),\left(f, b_{22}\right),\right. \\
\left(f, b_{23}\right),\left(f, B_{2}\right),\left(f, B_{2} B_{3}\right), \\
\left.\left(f, \Theta_{B}^{r e f}\right)\right\}\end{array}$ & 0.0984 \\
\hline$s \times B_{2}$ & $\begin{array}{c}\left\{\left(s, B_{2}\right),\left(s, B_{2} B_{3}\right),\left(s, \Theta_{B}^{r e f}\right),\right. \\
\left.\left(\Theta_{A}, B_{2}\right),\left(\Theta_{A}, B_{2} B_{3}\right),\left(\Theta_{A}, \Theta_{B}^{r e f}\right)\right\}\end{array}$ & $\begin{array}{c}\left\{\left(f, b_{22}\right),\left(f, b_{23}\right),\left(f, B_{2}\right)\right. \\
\left.\left(f, B_{2} B_{3}\right),\left(f, \Theta_{B}^{r e f}\right)\right\}\end{array}$ & 0.0504 \\
\hline$s \times B_{2} B_{3}$ & $\begin{array}{c}\left\{\left(s, B_{2} B_{3}\right),\left(s, \Theta_{B}^{r e f}\right)\right. \\
\left.\left(\Theta_{A}, B_{2} B_{3}\right),\left(\Theta_{A}, \Theta_{B}^{r e f}\right)\right\}\end{array}$ & $\begin{array}{c}\left\{\left(f, b_{22}\right),\left(f, b_{23}\right),\left(f, B_{2}\right),\right. \\
\left.\quad\left(f, B_{2} B_{3}\right),\left(f, \Theta_{B}^{r e f}\right)\right\}\end{array}$ & 0.0399 \\
\hline$s \times \Theta_{B}^{r e f}$ & $\left\{\left(s, \Theta_{B}^{r e f}\right),\left(\Theta_{A}, \Theta_{B}^{r e f}\right)\right\}$ & $\begin{array}{c}\left\{\left(f, b_{22}\right),\left(f, b_{23}\right),\left(f, B_{2}\right),\right. \\
\left.\quad\left(f, B_{2} B_{3}\right),\left(f, \Theta_{B}^{r e f}\right)\right\} \\
\end{array}$ & 0.0369 \\
\hline$f \times b_{22}$ & $\begin{array}{c}\left\{\left(f, b_{22}\right),\left(f, B_{2}\right),\left(f, B_{2} B_{3}\right),\right. \\
\left(f, \Theta_{B}^{r e f}\right),\left(\Theta_{A}, b_{22}\right),\left(\Theta_{A}, B_{2}\right), \\
\left.\left(\Theta_{A}, B_{2} B_{3}\right),\left(\Theta_{A}, \Theta_{B}^{r e f}\right)\right\}\end{array}$ & $\begin{array}{c}\left\{\left(f, b_{23}\right),\left(\Theta_{A}, b_{23}\right),\left(s, b_{22}\right),\right. \\
\left(s, b_{23}\right),\left(s, B_{2}\right),\left(s, B_{2} B_{3}\right), \\
\left.\left(s, \Theta_{B}^{r e f}\right)\right\}\end{array}$ & 0.1961 \\
\hline$f \times b_{23}$ & $\begin{array}{c}\left\{\left(f, b_{23}\right),\left(f, B_{2}\right),\left(f, B_{2} B_{3}\right),\right. \\
\left(f, \Theta_{B}^{r e f}\right),\left(\Theta_{A}, b_{23}\right),\left(\Theta_{A}, B_{2}\right), \\
\left.\left(\Theta_{A}, B_{2} B_{3}\right),\left(\Theta_{A}, \Theta_{B}^{r e f}\right)\right\}\end{array}$ & $\begin{array}{c}\left\{\left(f, b_{22}\right),\left(\Theta_{A}, b_{22}\right),\left(s, b_{22}\right),\right. \\
\left(s, b_{23}\right),\left(s, B_{2}\right),\left(s, B_{2} B_{3}\right), \\
\left.\left(s, \Theta_{B}^{r e f}\right)\right\}\end{array}$ & 0.1321 \\
\hline$f \times B_{2}$ & $\begin{array}{c}\left\{\left(f, B_{2}\right),\left(f, B_{2} B_{3}\right),\left(f, \Theta_{B}^{r e f}\right)\right. \\
\left.\left(\Theta_{A}, B_{2}\right),\left(\Theta_{A}, B_{2} B_{3}\right),\left(\Theta_{A}, \Theta_{B}^{r e f}\right)\right\}\end{array}$ & $\begin{array}{c}\left\{\left(s, b_{22}\right),\left(s, b_{23}\right),\left(s, B_{2}\right),\right. \\
\left.\left(s, B_{2} B_{3}\right),\left(s, \Theta_{B}^{r e f}\right)\right\}\end{array}$ & 0.0406 \\
\hline$f \times B_{2} B_{3}$ & $\begin{array}{c}\left\{\left(f, B_{2} B_{3}\right),\left(f, \Theta_{B}^{r e f}\right),\right. \\
\left.\left(\Theta_{A}, B_{2} B_{3}\right),\left(\Theta_{A}, \Theta_{B}^{r e f}\right)\right\}\end{array}$ & $\begin{array}{c}\left\{\left(s, b_{22}\right),\left(s, b_{23}\right),\left(s, B_{2}\right)\right. \\
\left.\left(s, B_{2} B_{3}\right),\left(s, \Theta_{B}^{r e f}\right)\right\}\end{array}$ & 0.0232 \\
\hline$f \times \Theta_{B}^{r e f}$ & $\left\{\left(f, \Theta_{B}^{\text {ref }}\right),\left(\Theta_{A}, \Theta_{B}^{\text {ref }}\right)\right\}$ & $\begin{array}{c}\left\{\left(s, b_{22}\right),\left(s, b_{23}\right),\left(s, B_{2}\right),\right. \\
\left.\left(s, B_{2} B_{3}\right),\left(s, \Theta_{B}^{r e f}\right)\right\} \\
\end{array}$ & 0.0182 \\
\hline$\Theta_{A} \times b_{22}$ & $\begin{array}{c}\left\{\left(\Theta_{A}, b_{22}\right),\left(\Theta_{A}, B_{2}\right)\right. \\
\left.\left(\Theta_{A}, B_{2} B_{3}\right),\left(\Theta_{A}, \Theta_{B}^{r e f}\right)\right\}\end{array}$ & $\left\{\left(s, b_{23}\right),\left(f, b_{23}\right)\right\}$ & 0.0887 \\
\hline$\Theta_{A} \times b_{23}$ & $\begin{array}{c}\left\{\left(\Theta_{A}, b_{23}\right),\left(\Theta_{A}, B_{2}\right)\right. \\
\left.\left(\Theta_{A}, B_{2} B_{3}\right),\left(\Theta_{A}, \Theta_{B}^{r e f}\right)\right\}\end{array}$ & $\left\{\left(s, b_{22}\right),\left(f, b_{22}\right)\right\}$ & 0.1054 \\
\hline$\Theta_{A} \times B_{2}$ & $\left\{\left(\Theta_{A}, B_{2}\right),\left(\Theta_{A}, B_{2} B_{3}\right),\left(\Theta_{A}, \Theta_{B}^{r e f}\right)\right\}$ & $\{\emptyset\}$ & 0.0072 \\
\hline$\Theta_{A} \times B_{2} B_{3}$ & $\left\{\left(\Theta_{A}, B_{2} B_{3}\right),\left(\Theta_{A}, \Theta_{B}^{r e f}\right)\right\}$ & $\{\emptyset\}$ & 0.0022 \\
\hline$\Theta_{A} \times \Theta_{B}^{r e f}$ & $\left\{\left(\Theta_{A}, \Theta_{B}^{r e f}\right)\right\}$ & $\{\emptyset\}$ & 0.0006 \\
\hline
\end{tabular}

